\title{
Joint Power and Subchannel Allocation in Relay Aided Multi-Cell OFDMA Networks
}

\author{
Huili Cheng, Qinghai Yang, Fenglin Fu \\ State Key Lab. of ISN, School of Telecomm. \\ Engineering, Xidian University, No.2 Taibainan-lu, Xi'an, \\ 710071, Shaanxi,China. Email: qhyang@xidian.edu.cn
}

\author{
Kyung Sup Kwak \\ Graduate School of IT and Telecomm., \\ Inha University, \#253 Yonghyun-dong, Nam-gu, \\ Incheon, 402-751, Korea. Email: kskwak@inha.ac.kr
}

\begin{abstract}
In this paper, a joint power and subchannel allocation strategy is investigated for relay-aided multi-cell OFDMA networks. The problem is formulated for maximizing the network throughput with minimal bandwidth cost under the users' minimum rate constraints. The method of Benders' Decomposition is employed to decompose the complicated problem into two subproblems, which solves the subchannel and power allocation issues separately. The low-complexity algorithms for two subproblems are developed to allocate the subchannel and power adaptively. Simulation results show that the algorithm converges and benefits higher throughput and spectral efficiency.
\end{abstract}

Index Terms-OFDMA, relay, resource allocation, Benders' decomposition.

\section{INTRODUCTION}

The rapid growing of wireless services requires the cell network can provide high-speed communication rate. Through adaptively allocating subchannel and power, the relay aided OFDMA system can achieve large throughput [1] by relaying the base station (BS) signal to cell-edge users. Relay aided OFDMA systems are strong candidates for future generation (4G) wireless communications [2].

Future broadband wireless communication systems are expected to provide heterogeneous data rate services, as well as high spectrum efficiency in limited band. In the case of poor subchannels, it will demand more power to target the transmission rate-threshold, leading to poor power efficiency. Meanwhile, through minimizing the consumed spectrum, the system can shut down some subchannels and then alleviate the interference caused by the aggressive frequency reuse. Eventually, it improves the system performance.

Several relay protocols, such as decode-and-forward (DF) and amplify-and-forward (AF), have been proposed to facilitate relaying. Meanwhile, how to efficiently allocate resource also draws great attention for more complex scenarios, wherein severe interference may be introduced by relays.

Efforts to leverage the subchannel and power allocation have been done in many literatures [3]-[8]. An adaptive subchannel allocation scheme to exploit system capacity and

\footnotetext{
*This research was supported by the RCUK for the UK-China Science Bridges Project: R\&D on (B) $4 \mathrm{G}$ wireless mobile communications, the MKE, Korea, under the ITRC program supervised by the NIPA (NIPA-2011-C10901121-0001) and the Fundamental Research Funds for the Central Universities (JY10000901002, JY10000901020), NSF China (60832001, 61001127), the 111 Project (B08038), China.
}

multi-user fairness in relay aided OFDMA cooperative cellular networks was studied in [5]. Different users' rate requirement is considered in [6], wherein the dual decomposition method is taken to solve the subchannel allocation problem. However, the power average distribution is adopted as prerequisite in [5]-[6], their results are not optimal. By allocating the power and subchannel simultaneously, a joint resource allocation method is proposed in [7], but result in high complexity. Best effort resource allocation and scheduling for homogeneous users in DF OFDMA systems are studied for different system configurations in [8]-[9]. However, in practice, users are heterogeneous with different QoS requirements such as the minimum required data rate. Though the above allocation and scheduling mechanisms can realize the optimal allocation of network resources, the high complexity and/or the disregarding of the power allocation adaption make them less attractive for OFDMA-based relay networks.

In this paper, we formulate the scheduling and resource allocation problem for multi-cell OFDMA DF relaying systems. Large system throughput and high spectral efficiency are obtained, while the users' minimum rate demand is satisfied. The Benders' Decomposition [10] method has been employed to transform the complex problem into two subproblems. Through iterating the results of the two subproblems [11], we can finally achieve an optimal solution.

The rest of the paper is organized as follows: Section II provides the system model. Section III formulates the resource allocation problem. Section IV reformulates the problem into a mixed integer linear optimization one. A iterative resource allocation algorithm with low complexity is proposed in Section V. Simulation results are given and discussed in section VI. Finally, Section VII concludes with a brief summary.

\section{SySTEM MODEL}

We consider a multi-cell OFDMA DF half-duplex relay networks with $K$ cells. Each cell, as shown in Fig.1, is equipped with one BS, $R$ relay stations (RS) and $M$ single-antenna users (UE). The maximum transmit power of $\mathrm{BS}$ and RS are $P_{B S}^{\max }$ and $P_{R S}^{\max }$, respectively. We assume the aggressive frequency reuse is employed and the total system bandwidth $W$ is divided into $N$ subchannels. Then the bandwidth of each channel is $B=W / N$. In this paper, we focus on the resource allocation and scheduling for heterogeneous users who need the help of 
relays. i.e. cell edge users in the shaded region in Fig.1. There is no direct link between BS and users for heavy blockage and long distance. Each relay only connects to one center BS and each user $m \in\left\{\begin{array}{lll}1 & 2 \ldots M\end{array}\right\}$ is served by one relay $r \in\left\{\begin{array}{llll}1 & 2 \ldots R\end{array}\right\}$ and its corresponding BS $k \in\left\{\begin{array}{llll}1 & 2 \ldots K\end{array}\right\}$. The downlink transmission from BS to users via the RS is accomplished in two steps:

- BS transmits and RS receives;

- RS decodes the previously received signals and forwards to the corresponding users.

In the paper, we assume perfect channel state information (CSI) is known at BS and the channel $n \in\left\{\begin{array}{lll}1 & 2 \ldots N\end{array}\right\}$ is timeinvariant within each frame. Here we further assume that intracell interference can be avoided by the orthogonal channel usage in a cell. There is only inter-cell interference due to the aggressive frequency reuse.

Let $p_{k, n}^{r}$ denote the transmit power of BS $k$ to the RS $r$ over subchannel $n . h_{k, n}^{r}$ represents the subchannel gains between BS $k$ and RS $r$ and $N_{0}$ is the noise power spectral density. Based on the Shannon formula, the transmission rate between $\mathrm{BS} k$ and the RS $r$ on subcarrier $n$ is given by

$$
R_{k, n}^{r}=B \log _{2}\left(1+\frac{p_{k, n}^{r}\left|h_{k, n}^{r}\right|^{2}}{I_{k 1}+B * N_{0}}\right),
$$

where $I_{k 1}$ is the received multi-cell interference on subcarrier $n$. Similarly, the transmission rate between RS $r$ and UE $m$ on subchannel $n$ is

$$
R_{m, n}^{r}=B \log _{2}\left(1+\frac{p_{m, n}^{r}\left|h_{m, n}^{r}\right|^{2}}{I_{k 2}+B * N_{0}}\right),
$$

where variables $p_{m, n}^{r}, h_{m, n}^{r}, I_{k 2}$ are defined in similar as the corresponding variables for the BS-RS links except that the signalling direction is from relay $r$ to user $m$.

For simplicity, we assume that the RS forwards the received data to the user on the same subcarrier with BS. Therefore the data rate on subchannel $n$ for user $m$ relayed by RS $r$ is

$$
R_{k, m, n}^{r}=\frac{1}{2} \min \left(R_{k, n}^{r}, R_{m, n}^{r}\right) .
$$

\section{PROBLEM FORMULATION}

In the two-hop scenario, the rate is dominated by the link with lower capacity. However, in practice, users locate at random positions and occupy a non-line-of-sight $(N L O S)$ communication link. Yet, a strong line-of-sight $(L o S)$ propagation channel is expected between the BS and the RS, since they are placed in relatively high positions and the blockages between them are limited. Besides, inter-cell interference is also relatively small in the BS-RS links. So as show in equation (3), we assume the RS-UE links have the dominated influence on the transmission rate

$$
R_{k, m, n}^{r}=\frac{1}{2} R_{m, n}^{r} .
$$

In this article, BS power is assumed to be allocated equally and we mainly focus on allocating the subchannel and RS power. Given by the user's minimum rate, the optimization

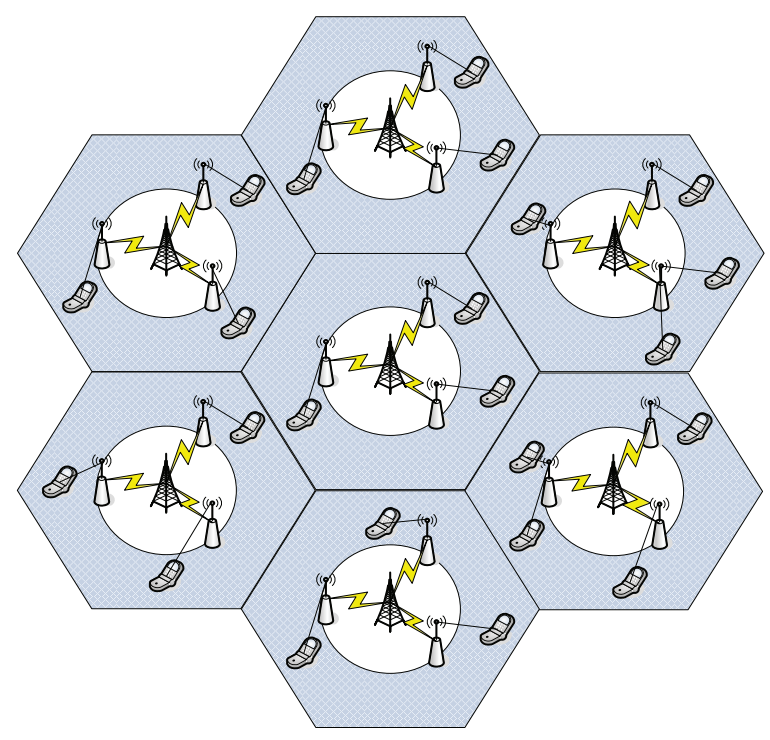

Fig. 1. The model of relay aided cellular network

problem of joint power and subcarrier allocation in cell $k$ can be formulated as follows:

$$
\begin{array}{ll} 
& \max \sum_{r=1}^{R} \sum_{m=1}^{M} \sum_{n=1}^{N} x_{m, n}^{r} R_{m, n}^{r} \\
\text { s.t. } \quad & 0 \leq \sum_{m=1}^{M} \sum_{n=1}^{N} x_{m, n}^{r} P_{m, n}^{r} \leq P_{R S}^{\max } \\
& \sum_{r=1}^{R} \sum_{n=1}^{N} x_{m, n}^{r} R_{m, n}^{r} \geq R_{m}^{\min } \quad \forall m \\
& 0 \leq P_{m, n}^{r} \leq x_{m, n}^{r} P_{m, n}^{r} \\
& \sum_{r=1}^{R} \sum_{m=1}^{M} \sum_{n=1}^{N} x_{m, n}^{r} \leq N \\
& \sum_{r=1}^{R} \sum_{m=1}^{M} x_{m, n}^{r} \leq 1 \quad x_{m, n}^{r} \in\{0,1\} .
\end{array}
$$

Here, $x_{m, n}^{r}$ is the allocation index, which equals to 1 if the subchannel $n$ is allocated to user $m$ through relay $r$, otherwise is 0 . Problem (5) aims at maximizing the cell $k$ 's total throughput. Constraint (6) limits the total power. (7) guarantees the users' minimize rate requirement. (8) ensure that $P_{m, n}^{r}$ is equal 0 if the sub-channel is not used. (9) gives the subchannel limitation and (10) states that the same channel can only be occupied by one user in each cell at the same slot. Solving problem (5), we can obtain the optimal subchannel and power allocation, which can achieve the highest throughput. However, we should notice that the optimal allocation may not be unique, since different association strategy may result in the same policy. The solution set of the problem (5) can be denoted as:

$$
\Omega=\{(X, P) \mid \forall(X, P) \in(5)\}
$$

In order to reduce the band consumption, it is desirable to 
find one solution $(X, P)$ in $\Omega$ that consumes the minimum numbers of subchannel. This can be achieved by solving the following problem:

$$
\begin{array}{ll} 
& \min \sum_{r=1}^{R} \sum_{m=1}^{M} \sum_{n=1}^{N} x_{m, n}^{r} \\
\text { s.t. } & \sum_{r=1}^{R} \sum_{m=1}^{M} \sum_{n=1}^{N} x_{m, n}^{r} \leq N \\
& \sum_{r=1}^{R} \sum_{m=1}^{M} x_{m, n}^{r} \leq 1 \quad x_{m, n}^{r} \in[0,1]
\end{array}
$$

By solving the problem (12), we can obtain a solution $(X, P)$, which has a minimum band requirment. According to the analysis, through solving problem (5) and problem (12) sequentially, we can simultaneously maximize the throughput and minimize the band consumption. Unfortunately, the problem (5) is a mixed-integer non-convex problem, thus it is difficult to find the global optimal solution. In the next section, we will transform the problems and adopt the method of Benders's Decomposition [11] to solve the joint problems

\section{Problem Translation}

We reformulate the problem (5) and (12) into a single optimization problem. By doing so, the two mixed integer nonconvex optimization problems can be simplified as a mixed integer linear optimization problem.

$$
\begin{aligned}
& \max \Phi(X, P) \\
= & \xi \sum_{r=1}^{R} \sum_{m=1}^{M} \sum_{n=1}^{N} R_{m, n}^{r}+(1-\xi) \sum_{r=1}^{R} \sum_{m=1}^{M} \sum_{n=1}^{N}\left(1-x_{m, n}^{r}\right) \\
\text { s.t. } \quad & 0 \leq \sum_{m=1}^{M} \sum_{n=1}^{N} x_{m, n}^{r} P_{m, n}^{r} \leq P_{R S}^{\max } \\
& \sum_{r=1}^{R} \sum_{n=1}^{N} x_{m, n}^{r} R_{m, n}^{r} \geq R_{m}^{m i n} \quad \forall m \\
& 0 \leq P_{m, n}^{r} \quad \forall \\
& \sum_{r=1}^{R} \sum_{m=1}^{M} \sum_{n=1}^{N} x_{m, n}^{r} \leq N \\
& \sum_{r=1}^{R} \sum_{m=1}^{M} x_{m, n}^{r} \leq 1 \\
& 0 \leq \xi \leq \frac{1}{\sum_{m=1}^{M} R_{m}^{r e q}+1}
\end{aligned}
$$

Here $\xi$ is a constant value. Referring to the disposing method of paper [11], the problem (13) is equivalent to maximize the whole throughput and simultaneously minimize the band consumption under the given user rate requirement. Then we can exploit the Benders' Decomposition method to solve the problem (13).

Lemma1: The model can use the method of Benders' Decomposition.
Proof: According to paper [10], we can definitely discover that the problem (13) satisfies the condition of the Benders' Decomposition required, which means the problem can be disposed by the method. Meanwhile, referring to its application, we can find the similar model used in a different context and then expand it to solve the above problem. The detailed proof is omitted for page limited.

\section{JOINT Optimization SOLUTION}

In this section, we resort the Benders' Decomposition to solve the problem. The key characteristic of the algorithm is that it divides the problem into two subproblems: a programming problem and a linear programming problem. Then in a finite number of iteration between the two subproblems, we can obtain the optimal value $(X, P)$.

\section{A. Benders' Decomposition}

The problem (13) is a union problem. It can be decomposed into a master problem:

$$
\begin{array}{ll} 
& \max \Phi(X, P) \\
& =\xi \mathrm{V}(X)+(1-\xi) \sum_{r=1}^{R} \sum_{m=1}^{M} \sum_{n=1}^{N}\left(1-x_{m, n}^{r}\right) \\
\text { s.t. } \quad & \sum_{r=1}^{R} \sum_{m=1}^{M} \sum_{n=1}^{N} x_{m, n}^{r} \leq N \\
& \sum_{r=1}^{R} \sum_{m=1}^{M} x_{m, n}^{r} \leq 1 \quad x_{m, n}^{r} \in\{0,1\} \\
& X=\left[x_{m, n}^{r}\right]
\end{array}
$$

and a subproblem:

$$
\begin{array}{ll} 
& \mathrm{V}(X)=\min \sum_{r=1}^{R} \sum_{m=1}^{M} \sum_{n=1}^{N} R_{m, n}^{r} \\
\text { s.t. } \quad & 0 \leq \sum_{m=1}^{M} \sum_{n=1}^{N} x_{m, n}^{r} P_{m, n}^{r} \leq P_{R S}^{\max } \\
& \sum_{r=1}^{R} \sum_{n=1}^{N} x_{m, n}^{r} R_{m, n}^{r} \geq R_{m}^{\min } \quad \forall m \\
& 0 \leq P_{m, n}^{r}
\end{array}
$$

Primarily, the problem (13) is divided into a subchannel allocation problem and a power allocation problem. It can be solved by exhaustive search and iteration. However, this will fail if particular $\mathrm{X}$ value results in an empty solution to problem (16).

\section{B. The Algorithm}

In this subsection, we introduce an algorithm to solve the problem. First, we dispose the (16) by the dual method. Then, by solving the dual of (16) in each iteration, it can generate optimality cut and feasibility cut to shrink the feasible region of (15). In particular, an optimality cut is added to (15) when the subproblem (16) is feasible and a feasible cut is added otherwise. Further, we rewrite the two subproblems as: 
Master problem:

$$
\begin{aligned}
\Phi= & \max \xi \theta+(1-\xi) \sum_{r=1}^{R} \sum_{m=1}^{M} \sum_{n=1}^{N}\left(1-x_{m, n}^{r}\right) \\
\text { s.t. } \quad & h\left(X, \alpha_{p}^{(i)}, \beta_{p}^{(i)}\right) \geq \theta \quad \forall i=1, \cdots S_{1} \\
\text { or } \quad & h\left(X, \alpha_{l}^{(j)}, \beta_{l}^{(j)}\right) \geq 0 \quad \forall j=1, \cdots S_{2} \\
& \sum_{r=1}^{R} \sum_{m=1}^{M} \sum_{n=1}^{N} x_{m, n}^{r} \leq N \\
& \sum_{r=1}^{R} \sum_{m=1}^{M} x_{m, n}^{r} \leq 1 \quad x_{m, n}^{r} \in[0,1] \\
& \theta \geq 0
\end{aligned}
$$

Subproblem:

$$
\begin{aligned}
h(X)= & \max (X, \alpha, \beta) \\
= & \max \sum_{r=1}^{R} \sum_{m=1}^{M} \sum_{n=1}^{N} R_{m, n}^{r}+\sum_{r=1}^{R}\left(-\alpha_{r} \sum_{m=1}^{M} \sum_{n=1}^{N} x_{m, n}^{r} P_{m, n}^{r}\right) \\
& +\sum_{m=1}^{M} \beta_{m}\left(\sum_{r=1}^{R} \sum_{n=1}^{N} R_{m, n}^{r}-R_{m}^{m i n}\right) \\
\text { s.t. } \quad & 0 \leq P_{m, n}^{r} \quad \forall m, n, r \\
& \vec{\alpha}=\left(\alpha_{r}\right) \geq 0 \quad \vec{\beta}=\left(\beta_{m}\right) \geq 0
\end{aligned}
$$

where $S_{1}+S_{2}=k, k$ is the iteration step. We resort to Benders' Decomposition method and it works as follows: firstly, starting with an initialization $X^{0}=\mathbf{0}$. It checks whether the subproblem (18) is bounded or not. If it is, add an optimality cut $h\left(X, \alpha_{p}^{(i)}, \beta_{p}^{(i)}\right) \geq \theta$ to master problem and takes it as a constraint condition, if (18) is unbounded, add a feasibility cut $h\left(X, \alpha_{l}^{(j)}, \beta_{l}^{(j)}\right) \geq 0$ to mater problem constraint condition where $\left(\alpha_{l}^{(j)}, \beta_{l}^{(j)}\right)$ is any point on the extreme ray for $X^{0}$. With the newly added constraint conditions, we can solve the problem (17) and update $X^{0}=X^{1}$. Then iteration continues until it converges.

The $k t h$ iteration object values of problem (18) and problem (17) are denoted as $\theta^{k}$ and $\Phi^{k}$ respectively. Here $\Phi^{k-1}=$ $\xi \theta^{k}+(1-\xi) \sum_{r=1}^{R} \sum_{m=1}^{M} \sum_{n=1}^{N}\left(1-x_{m, n}^{r(k-1)}\right)$

Lemma 2: The algorithm can converge to a global optimal solution

Proof: Following from the theories about the Benders' Decomposition [10] and related application, the algorithm is convergent and we can get global optimal solution to problem (13) with finite number of iterations.

In summary, the dynamic iteration procedure that is developed for the resource allocation is shown in following:

Step 1: set $\Phi^{0}=0, X^{0}=\mathbf{0}$ and $\mathrm{k}=1$

Step 2: Solve the problem (18) to obtain $h\left(X, \alpha_{p}, \beta_{p}\right) \geq$ $\theta$ or $h\left(X, \alpha_{l}, \beta_{l}\right) \geq 0$

Step 3: Take it as constraint condition as to solve problem (17) yield a new $\mathrm{X}^{k}$

Step 4: Calculate $\Phi^{k}-\Phi^{k-1}$. If $\Phi^{k}-\Phi^{k-1}=0$ stop, else $\mathrm{k}=\mathrm{k}+1$,go to step 2
Step 5: Compute the optimal power allocation $P^{*}$, then $\left(X^{k}, P^{*}\right)$ is the optimal solution to problem (13)

\section{Simulation}

\section{A. Simulation Models and Parameters}

In the simulation, we show the validity and availability of the algorithm. Here we consider a low mobility wireless OFDMA cellular system. The user uniformly distributed in the relay area. The relay stations, as shown in fig.1, are equally distributed at the inner cell boundary of each cell for assisting the transmission. To simplify the channel gains, we calculate it as $h_{m, n}^{r}=d_{m, r}^{-4}$, where $d_{m, r}$ is the distance between RS and users. The concrete parameters are given in TABLE 1:

TABLE 1 PARAMETERS

\begin{tabular}{|l|l|}
\hline Parameter & value \\
\hline Carrier frequency & $f=2 \mathrm{GHz}$ \\
\hline System bandwidth & $\mathrm{W}=5 \mathrm{MHz}$ \\
\hline Number of subchannel & 100 \\
\hline Band of subchannel & $50 \mathrm{khz}$ \\
\hline Cellular layout & Hexagonal grid, 7 cell site \\
\hline Cell radius & $1200 \mathrm{~m}$ \\
\hline Distance BS to RS & $800 \mathrm{~m}$ \\
\hline Relay area & $800-1200 \mathrm{~m}$ \\
\hline RS total power & $43 \mathrm{dBm}$ \\
\hline Thermal noise density & $-174 \mathrm{dBm} / \mathrm{Hz}$ \\
\hline Subchannel gain & $d_{i j}^{-4}$ \\
\hline Relay users number per cell & $10 / 15 / 20 / 25 / 30$ \\
\hline Relay number & 3 \\
\hline User rate demand & $100 \mathrm{~kb} / \mathrm{s}$ \\
\hline$\xi$ & $1 /(1 e 6)$ \\
\hline
\end{tabular}

\section{B. Simulation Results and Discussions}

In this section, we evaluate the proposed algorithm to the one proposed in [5], which take the power average allocation as the precondition. The algorithm first allocates subchannel to satisfy users' rate requirement. Then it allocates the subchannel to the user who can produce the maximum rate. In order to accelerate the convergent speed, we set the algorithm convergence, when $\Phi^{k}-\Phi^{k-1}=\sigma$. Here $\sigma=1$.

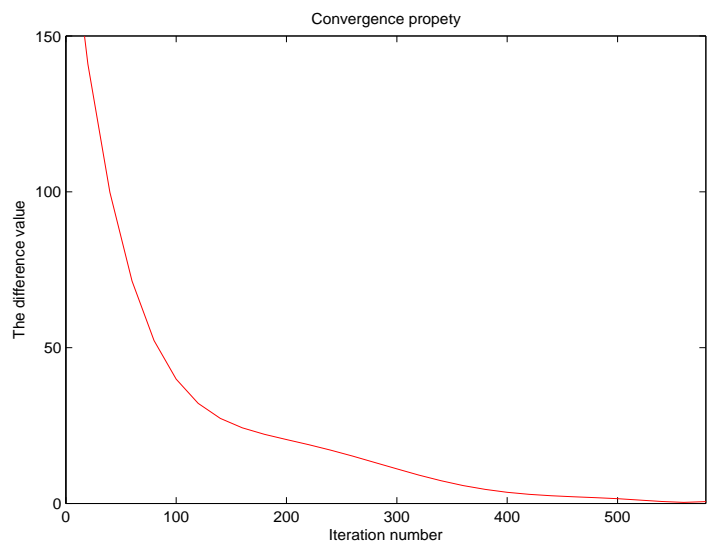

Fig. 2. Convergence property of the algorithm under the user number is 20 


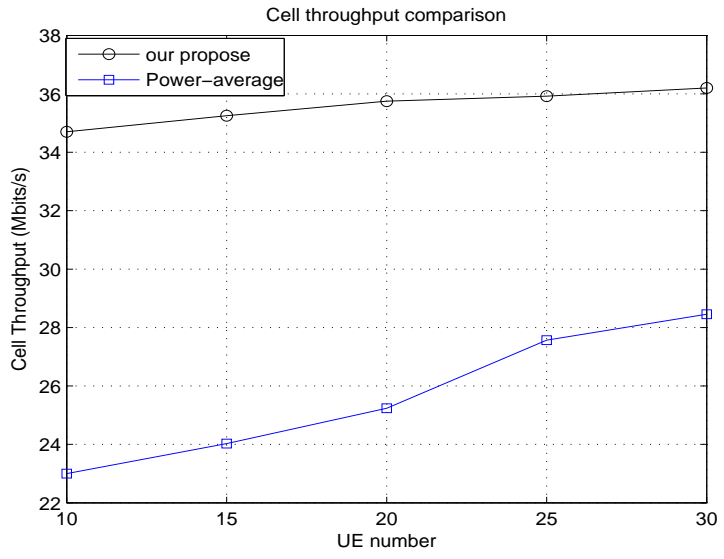

Fig. 3. Throughput vs. the number of users

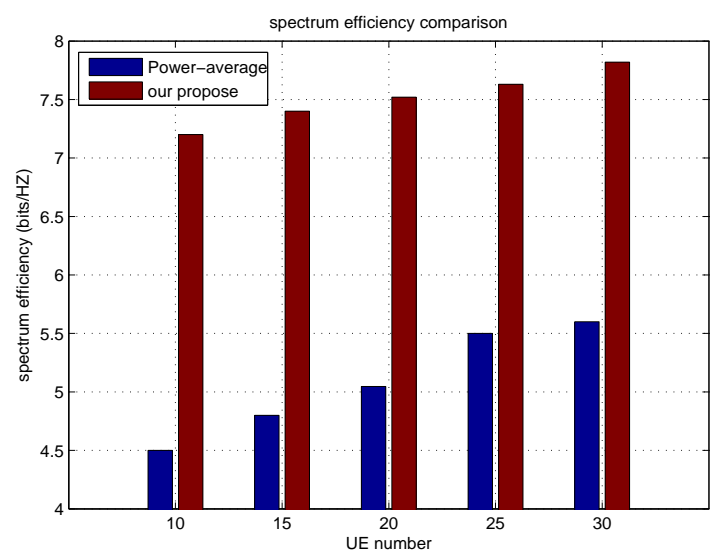

Fig. 4. Spectral efficiency vs. the number of users

Fig.2 illustrates the convergence property with 20 users. The different value is $\Phi^{k}-\Phi^{k-1}$. We can easily observe that the algorithm converges. At the beginning, it takes a quick shrink. With the iteration number increase, the convergence speed becomes slower. At last, it reaches a steady value.

Next, we shall compare our proposed algorithm with the traditional power average allocation algorithm [5] from the perspectives of system throughput. Two scenarios of different number of users are considered. And we assume each user has a minimum rate requirement of 100kbps. As shown in Fig.3, our proposed algorithm provides higher system throughput. In contrast to the traditional one, we deploy the power adaptively and acquire the optimal solution by plenty of iteration steps. So we can improve the system throughput to a certain extent. When the user number is 10 , the improvement is nearly $10 \mathrm{Mbps}$, this implies that the adaptive allocation contributes greatly to enhance system capacity. Fig.4 depicts the spectrum efficiency of the two algorithms. As expected, the proposed algorithm shows better results.

\section{CONCLUSION}

In this paper, we proposed a subchannel and power allocation strategy in the relay aided multi-cell OFDMA networks. By combining two optimization problems into a single optimization problem, we simultaneously achieve maximum throughput and minimize the band consumption. With Benders' Decomposition, the problem was then decomposed into a master problem focusing on subchannel allocation and a subproblem on power allocation. The algorithm was proposed to obtain the optimal solutions of subchannel and power allocations. Simulation results showed that the proposed approach achieves higher throughput and higher spectral efficiency than the traditional ones.

\section{REFERENCES}

[1] Daniel. C, Schultz, and B. Walke, "Fixed relays for cost efficient 4G network deployments," in Proc. PIMRC'07, Sept. 2007.

[2] Nokia Siemens Networks, "The advanced LTE toolbox for more efficient delivery of better user experience," www.nokiasiemensnetworks.com, March 2011

[3] M. Awad and X. Shen, "Ofdma based two-hop cooperative relay network resources allocation," in Communications, 2008. ICC '08. IEEE International Conference on May 2008, pp. 4414-4418

[4] Z. Shen, X. Wang, and H. Zhang, "Power allocation and subcarrier pairing for ofdm-based af cooperative diversity systems," in Vehicular Technology Conference, 2009, pp. 1-6, Dec. 2006

[5] Nidhal Odeh, Mehran Abolhasan, and Farzad Safaei, "Low Complexity Interference Aware Distributed Resource Allocation for Multi-cell OFDMA Cooperative Relay Networks," Wireless Communications and Networking Conference (WCNC), 2010 IEEE, pp 978-1-4244-6396-1.

[6] Danhua Zhang, Youzheng Wang, Jianhua Lu, "QoS Aware Relay Selection and Subcarrier Allocation in Cooperative OFDMA Systems," IEEE Communications letters,vol. 14, No. 4, April 2010, pp 1089-7798

[7] Derrick Wing Kwan Ng and Robert Schober, "Resource Allocation and Scheduling in Multi-Cell OFDMA Decode-and-Forward Relaying Networks." Wireless Communications, IEEE Transactions, Volume. 10, Issue.7, pp 2246 - 2258 July 2011.

[8] Y. Cui, V. K. N. Lau, and R. Wang, Distributive Subband Allocation,Power and Rate Control for Relay-Assisted OFDMA Cellular System with Imperfect System State Knowledge, "IEEE Trans. Wireless Commun, vol. 8, pp. 5096 C 5102, Oct 2009.

[9] L. Weng and R. D. Murch, Cooperation Strategies and Resource Allocations in Multiuser OFDMA Systems, IEEE Trans. Veh. Technol, " IEEE Trans. Veh. Technol., vol. 58, pp. 2331 C 2342, Jun 2009.

[10] J. Benders, "Partitioning procedures for solving mixed-variables programming problems," in Numerische Mathematik, vol.4, pp. 238-252, April 1962.

[11] Jieying Chen, Li Ping Qian, "On Optimization of Joint Base Station Association and Power Control via Benders' Decomposition," Global Telecommunications Conference 2009, pp 978-1-4244-4148-8, Nov. 30 2009-Dec. 42009 